\title{
APLICACIÓN DE LA LÓGICA DIFUSA EN PROYECTOS
}

\author{
Application of Fuzzy Logic in projects
}

\section{EPISTEMUS}

ISSN: 2007-8196 (electrónico)

ISSN: 2007-4530 (impresa)

Víctor Antonio Entenza Boggiano 1

Lucía Argüelles Cortés ${ }^{2}$

Daylenis Dorta Enríquez ${ }^{3}$

Recibido: 12 de septiembre de 2018, Aceptado: 30 de noviembre del 2018

Autor de Correspondencia:

Víctor Antonio Entenza Boggiano

Correo:ventenza@uclv.cu

\section{Resumen}

Este trabajo se propuso brindar una pauta metodológica para utilizar las técnicas de la lógica difusa en la gestión de proyectos. Primeramente, se introdujeron las nociones básicas sobre las cuales se fundamenta la lógica difusa requerida. Se focalizaron tres aspectos correspondientes al manejo de proyectos donde es posible aplicar la Lógica Difusa para evaluar con más objetividad su significación y alcance: el manejo de índices que justifican la racionalidad de la inversión, de manera que se puedan medir beneficios económicos; la determinación de una jerarquía de riesgos con vista a tomarlos en consideración para minimizar su impacto y la toma de decisión en un ambiente en el que confluyen factores tanto objetivos como subjetivos. Luego, fueron explicadas técnicas de la lógica difusa correspondientes: la utilización del VAN difuso, el análisis jerárquico de alternativas y la toma de decisión mediante un cierto consenso. Se discutieron las especificidades de tres problemas, el primero sobre determinación de VAN difuso relacionado con fondos de flujos netos generados en varios años, el segundo correspondiente a la determinación del riesgo más significativo para una empresa que desarrolla ciertos tipos de proyectos a los cuales están asociados tres riesgos y el último vinculado a la valoración de ofertas sobre la base de la creación de un perfil. Finalmente se propuso una metodología de trabajo en dichos aspectos para aplicar las técnicas correspondientes en la gestión de proyectos. Esta metodología viabiliza esta gestión, dotando al análisis de proyectos del tratamiento y rigor matemáticos requeridos para ambientes de incertidumbre.

Palabras clave: gestión de proyectos, índices difusos, toma de decisión por consenso, riesgos en proyectos, métodos jerárquicos.

\section{Abstract}

This work was proposed to provide a methodological guide to use the techniques of fuzzy logic in project management. First, the basic notions on which the required fuzzy logic is based were introduced. Three aspects were focused on the management of projects, where it is possible to apply Fuzzy Logic to evaluate with more objectivity its significance and scope: the management of indexes that justify the rationality of the investment, so that economic benefits can be measured; the determination of a hierarchy of risks with a view to taking them into consideration to minimize their impact and decision making in an environment in which both objective and subjective factors converge. Then, corresponding fuzzy logic techniques were explained: the use of diffuse VAN, the hierarchical analysis of alternatives and decision making through a certain consensus. The specificities of three problems were discussed, the first one regarding the determination of diffuse VAN related to funds of net flows generated in several years, the second corresponding to the determination of the most significant risk for a company that develops certain types of projects to which there are associated three risks and the last one linked to the valuation of offers based on the creation of a profile. Finally, a work methodology was proposed in these aspects to apply the corresponding techniques in project management. This methodology makes this management viable, providing the analysis of the mathematical treatment and rigor required for uncertainty environments.

Keywords: project management, fuzzy indexes, decision making by consensus, project risks, hierarchical methods.

1 Universidad Central "Marta Abreu" de Las Villas. Correo: ventenza@uclv.cu

2 Universidad Central "Marta Abreu" de Las Villas. Correo: largue@uclv.edu.cu

3 Universidad Central "Marta Abreu" de Las Villas. Correo: ddortae@uclv.cu 


\section{INTRODUCCIÓN}

La Lógica Difusa ha encontrado múltiples aplicaciones en las más variadas áreas del conocimiento a partir de su formalización en la década de los años 60 del siglo XX por parte del Profesor Lofti Zadeh. La misma se ha construido sobre una apropiada generalización de los conjuntos tradicionalmente conocidos (para los cuales la condición de pertenencia de cada uno de sus elementos constituye una proposición que es susceptible de ser cierta o de ser falsa de forma categórica) a conjuntos en los cuales la afirmación de pertenencia de elementos a este está afectada por cierta subjetividad; contribuyendo así al tratamiento matemático riguroso de aquellos sistemas para los cuales la información vaga o imprecisa es sustancial, y a la comprensión de dichos sistemas. De modo que esta teoría de los conjuntos difusos y la lógica difusa se hace posible de recurrir cuando no es posible valorar con precisión un conjunto de variables o situaciones.

Entre los casos en que se pueden presentar situaciones de imprecisión se tienen los asociados a la gestión de proyectos. Ejemplo de ello es un proyecto que pretende mejorar determinados elementos construidos. ¿Cómo se puede calificar la situación del objeto de análisis: muy buena, buena, regular, mala? ¿O el riesgo de una edificación frente a un sismo: muy alto, alto, mediano, bajo? Todas estas son calificaciones de desempeño, expresadas en términos lingüísticos, las cuales son posibles de estudiar con el uso de la teoría de lógica difusa. En ésta, el tránsito de una calificación a otra es gradual, no es drástico.

En la caracterización de las condiciones de un proyecto, se observan generalmente tres enfoques: la medición directa, la estimación aproximada y la valoración apreciativa. Por ejemplo, se puede decir:

1.El proyecto tiene una duración de cinco años (medición directa).

2. El costo del proyecto está entre 30000 y 40000 pesos (estimación aproximada).

3. El proyecto es de mediana complejidad (valoración apreciativa).

En los dos últimos enfoques, están presentes fuentes de incertidumbre debidas a imprecisiones en la información y a imprecisiones lingüísticas respectivamente.

La lógica difusa ha dado lugar a poderosos métodos, surgidos en la actualidad, que dan un tratamiento matemático a términos permeados de subjetividad, obteniéndose consecuentemente resultados mucho más rigurosos, satisfaciendo así, en gran parte, la necesidad de técnicas matemáticas para abordar la evaluación de las problemáticas que se presentan en los campos del conocimiento donde es usual el manejo de imprecisiones provenientes de características cualitativas subjetivas. Estas técnicas abordan problemas de riesgo, de toma de decisiones, etc., comunes a las ciencias exactas, las áreas

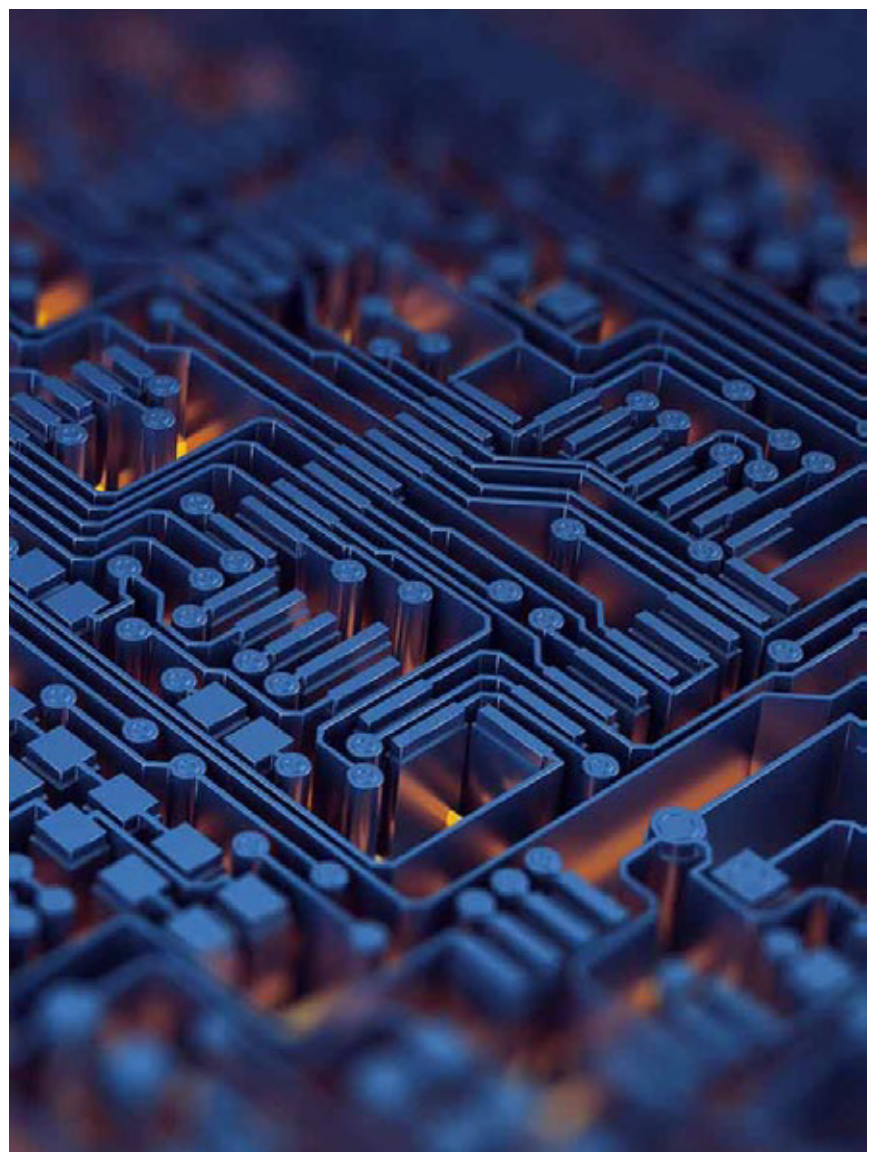

técnicas y aún a las ciencias humanísticas. Sin embargo, usualmente no son utilizadas en el campo de la gestión de proyectos. Por lo tanto, se hace necesario ofertar un conjunto de técnicas de la lógica difusa para ser tomadas en consideración en la realización y valoración de proyectos.

La incorporación de estas técnicas para llegar a la toma de decisiones en un consenso que eleve la calidad del proyecto es una forma muy actual de concebirlos y de llevarlos a ejecución, por lo que la familiarización con las mismas, conjuntamente con sus aplicaciones, debe constituir parte integral en el desarrollo de cada proyecto. De aquí que este trabajo se propone como objetivo brindar una pauta metodológica para utilizar las técnicas de la lógica difusa en la gestión de proyectos.

También en este trabajo se explica la importancia de estas técnicas y se presentan sugerencias de aplicación en este campo.

Con el objetivo de definir formalmente un conjunto borroso, observemos que un conjunto abrupto está caracterizado por la función característica $C(A)$, que es la función definida en el universo $X y$ con valores en el conjunto discreto $\{0,1\}$ que describe la condición de pertenencia de un elemento del universo al conjunto ordinario $A$. De aquí que resulta natural definir formalmente un "conjunto borroso" A mediante una función que llamaremos de pertenencia al conjunto $\mathrm{A}$ y denominaremos $f_{A}(\mathrm{x})$, la 
cual asigna a cada elemento de $\mathrm{X}$ un valor que estará comprendido en el intervalo [0,1]. Este valor responde a un cierto criterio fundamentado de manera apropiada y esta función representa el grado de pertenencia con que un elemento del universo $X$ pertenece $a$ un subconjunto $A$ de dicho universo.

En este sentido, la lógica difusa generaliza la lógica bivalente, resultando más efectiva en casos donde esta última no resulta adecuada, en particular en problemas donde están presentes diversos tipos de riesgo y la toma de decisiones mediante análisis que involucran varios criterios.

Un caso particular importante y usual de función de pertenencia viene dado como generalización del siguiente ejemplo.

Para estimar una tasa de interés anual, fue consultado un experto que informó que este valor se encontrará entre $\mathrm{a}=0.095$ y $c=0.15$, y que el valor al que más confianza puede asignársele es $b=0.12$. La confianza crece linealmente desde cero hasta uno para los valores ubicados entre el mínimo señalado y el más confiable y decrece linealmente desde uno hasta cero entre el más confiable y el máximo propuesto. En este caso, la representación gráfica del número borroso es un triángulo con vértices $a, b$, c. Debido a esta interpretación, los números representados por dos valores extremos y un valor intermedio significativo se llaman números triangulares y se denotan para su manejo mediante $[a ; b ; c]$.

Los números difusos triangulares simplifican el tratamiento de algunas operaciones tales como la adición, la diferencia, la multiplicación y la división entre ellos (Zadeh L., 1996).

\section{MATERIALES Y MÉTODOS O METODOLOGÍA COMPUTACIONAL}

A partir de las ideas presentadas anteriormente se define, como problema de investigación, cómo aplicar las herramientas de la lógica difusa en los problemas de gestión de proyectos. Así, el objeto de estudio de esta investigación está constituido por las técnicas matemáticas derivadas de la lógica difusa. Su campo de acción abarca el estudio de los conjuntos difusos o borrosos, los conceptos básicos de la lógica a la que estos dan lugar (lógica difusa o borrosa), la variedad de técnicas correspondientes a esta lógica. Su campo de aplicación consiste en aquellas situaciones problémicas de la gestión de proyectos asociadas a su valoración general, la toma de decisiones, la percepción y valoración del riesgo.

Al tener como objetivo (ya mencionado) brindar una pauta metodológica para utilizar las técnicas de la lógica difusa en la gestión de proyectos, el presente trabajo defiende la siguiente hipótesis: las técnicas derivadas de la lógica difusa constituyen una herramienta útil que debe ser tomada en consideración para la gestión de proyectos.

La ejecución de un proyecto puede ser considerada como una sucesión de toma de decisiones con el objetivo de resolver los problemas que se van sucediendo a lo largo de su puesta en práctica, debido a la necesidad de elegir

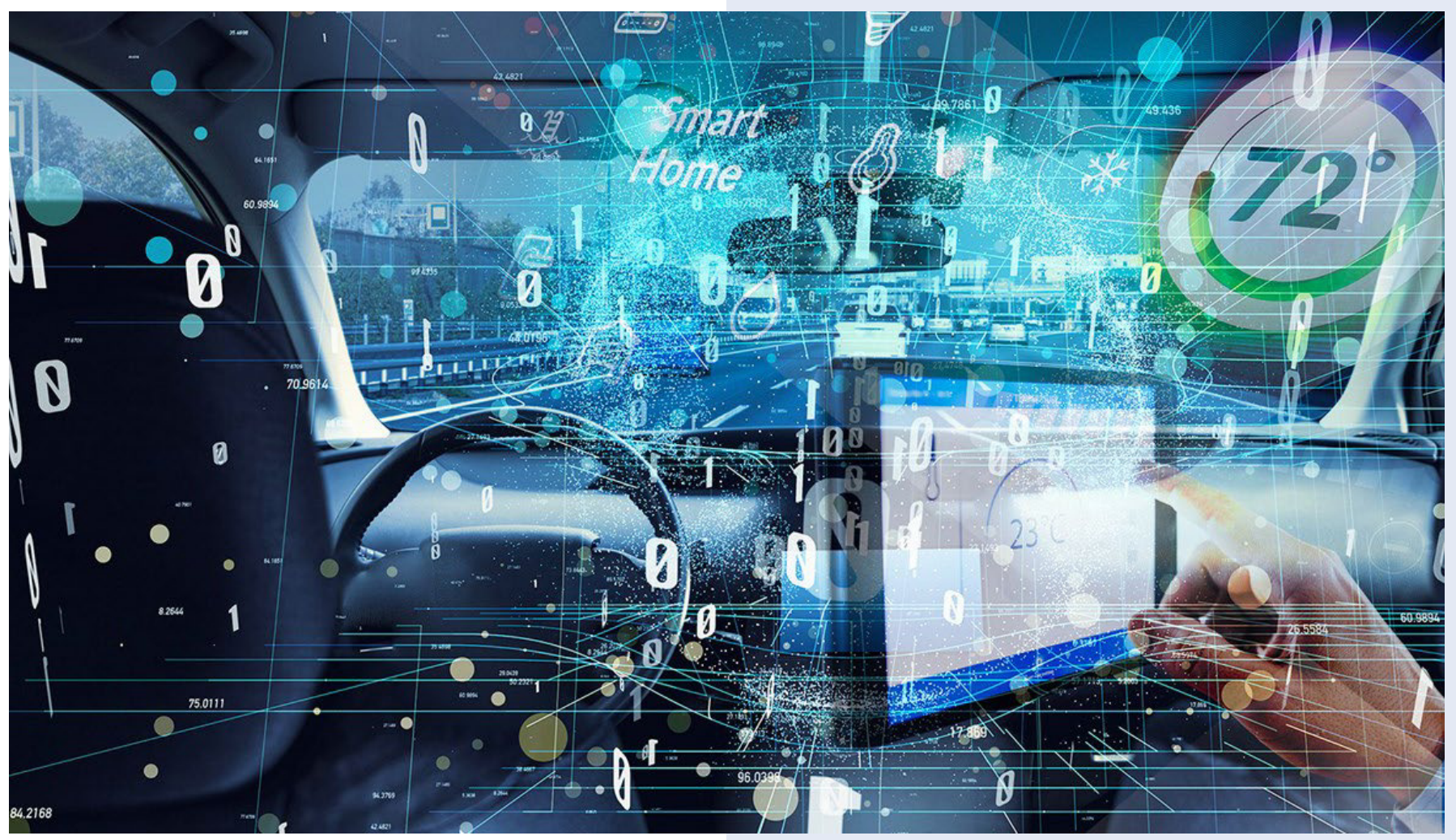


una solución entre varias propuestas para cada caso. Los problemas de toma de decisiones que se plantean son, en la mayoría de los casos, problemas multicriterio puesto que hay que tener en cuenta distintos criterios y puntos de vista que a menudo están en conflicto, siendo la decisión el resultado de un compromiso entre todos ellos.

Todo proceso de decisión transcurre en un contexto que se denomina ambiente o entorno.

El conjunto de características que define la situación de decisión respecto al entorno, puede ser de diversa naturaleza pudiéndose dar los siguientes casos:

Decisiones en ambientes de certidumbre: cuando se conocen con exactitud las consecuencias que conlleva la selección de cada alternativa.

$\bowtie$ Decisiones en ambientes donde interviene el azar: cuando se conoce una distribución de probabilidades de las consecuencias que tiene la selección de una determinada alternativa.

Decisiones en ambiente de incertidumbre o borrosos: cuando en la caracterización de las alternativas intervienen evaluaciones de carácter subjetivo.

La mayoría de las situaciones de decisión de la vida real tienen lugar en ambientes de incertidumbre en los que los objetivos, las restricciones y las consecuencias de las posibles actuaciones no son conocidos con precisión. El origen de tal imprecisión tiene, entre otras, las siguientes causas:

WInformación incuantificable. Por ejemplo: El precio de un nuevo producto puede ser fácilmente determinado a través de su costo de importación o de fabricación, mientras que sus atributos tales como la seguridad y la comodidad son siempre expresados en términos lingüísticos tales como bueno, aceptable, malo, etc. Se trata de datos cualitativos y, por lo tanto, sujetos a valoración subjetiva.

Información incompleta. Por ejemplo: La cuantía de una etapa del proyecto con requerimientos adicionales puede ser medida por algunos economistas como "alrededor de 25000 unidades monetarias". Tales datos pueden ser representados mediante números difusos pues la información es incompleta.

" Información imposible de obtener. Algunas veces la obtención de datos exactos se realiza a un coste muy elevado, pudiendo ser deseable obtener una "aproximación" a esos datos. También, cuando los datos son muy sensibles (secretos gubernamentales, datos bancarios, etc.), se suelen usar datos aproximados o descripciones lingüísticas. La información es difusa debido a su no disponibilidad.

$\rightarrow$ Ignorancia parcial. Cierta borrosidad es atribuida a la ignorancia parcial de un fenómeno a causa del desconocimiento de parte de los hechos, como es el caso del riesgo.

Los métodos clásicos de decisión han sido diseñados para tratar problemas de decisión en ambientes de certidumbre. Estos métodos presentan un serio inconveniente en cuanto a su aplicabilidad en entornos de incertidumbre. En estos ambientes no siempre es posible determinar los datos de partida exactos que requieren estos métodos y sólo es posible disponer de "aproximaciones" como "alrededor de cinco", "muy importante" o"entre seis y siete". Para tratar problemas de decisión de esta naturaleza, en los que los datos son imprecisos, vagos o borrosos, la Teoría de la Lógica Borrosa creada por Zadeh en 1965, se ha perfilado como una de las más adecuadas, debido a su capacidad para tratar conceptos vagos e imprecisos.

El concepto de riesgo, asociado a la idea de porvenir sin certeza, es propio de la sociedad humana y su consideración se remonta a las antiguas civilizaciones que realizaron sistemas de control de inundaciones para reducir el impacto de este suceso natural.

El análisis del riesgo condujo a la teoría de las probabilidades, la cual viabiliza la toma de decisiones bajo condiciones de riesgo e incertidumbre. Actualmente, debido al gran desarrollo de la ciencia y la tecnología existe la tendencia a la planificación, el pronóstico y la prevención conjuntamente con la mitigación para reducir riesgos. Por su enfoque, muchos de estos análisis no facilitan la toma de decisiones o no consideran aspectos multidisciplinares.

En particular, en ocasiones no se valora la información cualitativa proveniente de las imprecisiones y los matices del lenguaje.

\section{POSIBILIDADES DE APLICACIÓN DE TÉCNICAS DIFUSAS EN EL MANEJO DE PROYECTOS}

En este trabajo se focalizan tres aspectos en el manejo de proyectos donde es posible aplicar la Lógica Difusa para evaluar con más objetividad su significación y alcance. Estos aspectos se mencionan y detallan seguidamente.

\section{Manejo de índices para medir beneficios económicos}

En la evaluación de proyectos hay índices que justifican la racionalidad de la inversión. Las herramientas tradicionales pueden ser adaptadas a un contexto de incertidumbre mediante la utilización de números borrosos triangulares. Entre estos índices pueden mencionarse: el VAN (valor actual neto), el TIR (tasa interna de retorno), IR (índice de rentabilidad), etc. (Vostrov, 2014). En particular, el VAN expresa el incremento de riqueza, en unidades monetarias, que genera el proyecto. En condiciones de certeza, el proyecto se acepta si VAN $\geqq 0$. La fórmula para su cálculo es:

$$
\text { VAN }=-I_{0}+\sum_{t=0}^{n} \frac{F F N t}{(k)^{t}}
$$


Donde FFNt es el flujo de fondos neto correspondiente al período $t, k$ es la tasa de actualización $t$ es la medida de tiempo (por lo general en meses o en años), $I_{0}$ la inversión inicial de recursos que demanda el proyecto.

Por sus características conceptuales, tanto los flujos de fondos como la evolución de la tasa en períodos amplios admiten una representación mediante números difusos debido a la incertidumbre inherente a dichos valores. Desde este punto de vista, vamos a evaluar la marca de un producto que una empresa comercializa, con buena aceptación en el mercado y que tiene cinco años de vida útil.

\section{La determinación de una jerarquía de riesgos}

El riesgo se maneja mediante variables lingüísticas que asumen valores representados por conjuntos difusos, tales como: alto, bajo, medio, mayor.

En términos generales, se puede decir que el riesgo es un indicador de la ocurrencia de hechos que afectan el cumplimiento de los objetivos de un proyecto. La perspectiva del análisis del riesgo involucra cuatro orientaciones: identificación, reducción, transferencia (protección financiera) y manejo de consecuencias.

Conceptualmente, el riesgo está dado como una función de la amenaza y la vulnerabilidad. La amenaza es la posibilidad de un suceso potencialmente desastroso y la vulnerabilidad es la predisposición intrínseca de un grupo de elementos expuestos a sufrir una afectación. Por tanto, la amenaza es un peligro latente que puede afectar elementos vulnerables.

Los elementos en riesgo son de naturaleza muy variada: la población, edificaciones civiles, actividades económicas, servicios públicos, empresas productivas, etc.

De aquí que si se pretende la estimación del riesgo, constituye un paso previo el análisis y la evaluación de la amenaza como variable de fundamental importancia, así como también el estudio de la vulnerabilidad relacionada con la capacidad de los elementos expuestos para soportar la acción de los fenómenos.

La apreciación de la amenaza y de la vulnerabilidad puede ser muy diferente desde la óptica de técnicos evaluadores, comunidades afectadas y autoridades financieras, por lo que hoy se acepta que es necesario profundizar en la percepción individual y colectiva del riesgo para identificar las causas e investigar características que favorezcan la previsión y la mitigación del mismo.

Del análisis anterior se deriva que desde el punto de vista algorítmico, la metodología computacional que se vaya a utilizar debe descansar en la determinación de los elementos bajo riesgo como objeto de trabajo, la identificación de las amenazas como variables de entrada y la descripción de las vulnerabilidades asociadas que se corresponderán con los grados de afectación dados por las funciones de pertenencia.
Para el análisis del riesgo, el enfoque de las denominadas ciencias naturales concentra su estudio en el conocimiento de las amenazas. Por su parte, el enfoque de las ciencias aplicadas está dirigido hacia los efectos del suceso sobre los elementos expuestos a partir de la evaluación de la vulnerabilidad. Finalmente, el enfoque de las ciencias sociales ha hecho énfasis en la capacidad de las comunidades de absorber el impacto y en la recuperación después de un suceso. Consecuentemente, la especialización del tratamiento del riesgo en aspectos muy específicos no ha facilitado la visión integral y multidisciplinar del mismo que se requiere.

En particular, en las empresas productivas y de servicios, los riesgos que son objeto de interés pueden ser agrupados según sus procedencias en los tipos que se describen a continuación.

Inherentes: son propios de cada entidad laboral en dependencia de la actividad que realice.

Incorporados: aparecen como resultado de la conducta humana.

Administrativos: tienen lugar por fallas en el control del sistema de legislaciones estipuladas.

El objetivo primario del diagnóstico es determinar los factores de cada uno de estos tipos para realizar una evaluación consecuente.

En general, para establecer jerarquías, existen en la Lógica Difusa técnicas con diverso grado de análisis de su consistencia. Sin embargo para otros problemas es más idóneo aplicar alguna estrategia de optimización. (Díaz Córdova, Coba Molina, \& Navarrete, 2017) (Cruz Martínez, 2017)

\section{La toma de decisión}

En el ámbito de las ofertas de proyectos, se plantean problemas que exigen la toma de decisiones en un ambiente en el que confluyen factores tanto objetivos como subjetivos. Es por esto que la preparación de una decisión obedece más a criterios vagos que a criterios precisos, por lo que la realidad, permeada de imprecisión lingüística no puede estudiarse en términos absolutos con técnicas aplicables a situaciones ciertas ni aún aleatorias, pues en la búsqueda de la precisión intentando ajustar el mundo real a modelos matemáticos rígidos y estáticos se pierde información valiosa.

Con este enfoque, se pueden abordar los problemas de decisión en grupo que abarcan todos aquellos donde intervienen opiniones de expertos, necesidad de consenso, etc.

Un problema de Toma de Decisión en Grupo (TDG) se define como una situación de decisión caracterizada por los siguientes hechos:

(i) Hay dos o más expertos, cada uno de ellos con sus propias percepciones, actitudes, motivaciones y conocimiento. 
(ii) Los expertos reconocen la existencia de un problema común

(iii) Dichos expertos intentan obtener una decisión en común.

Para abordar el análisis que requiere tomar la mejor decisión, se han intentado diversas aproximaciones teóricas, algunas de ellas basadas en un conjunto de relaciones de preferencia establecidas mediante etiquetas lingüísticas (Ruvalcaba Coyaso \& Vermonden, 2015 ).

En algunos métodos, interviene de manera explícita la consideración de los diversos expertos y la importancia que a éstos se les concede. En otros métodos, el consenso de los expertos es preliminar a la aplicación de los mismos.

\section{ILUSTRACIÓN DE LA APLICACIÓN DE TÉCNICAS DIFUSAS EN EL ÁMBITO DE LOS PROYECTOS}

A continuación se ejemplifica la aplicación de algunas técnicas de la Lógica Difusa en los aspectos mencionados, se interpreta la base conceptual y se presenta la metodología de aplicación del método correspondiente.

\section{VAN difuso}

De acuerdo con los criterios de un experto, los fondos de flujos netos que generará en cada uno de los años serán:

$\mathrm{F} 1=[150000 ; 185000 ; 215000]$

$F 2=[160000 ; 200000 ; 230000]$

$\mathrm{F} 3=[173000 ; 205000 ; 230000]$

$\mathrm{F} 4=[175000 ; 210000 ; 232000]$

$\mathrm{F} 5=[180000 ; 212000 ; 235000]$

Las tasas de actualización estimadas son:

$K=[0.12 ; 0.15 ; 0.17]$ para los dos primeros años

$\mathrm{K}=[0.15 ; 0.18 ; 0.2]$ para los tres años restantes.

La fórmula VAN para números difusos quedará:

VAN $=-I_{0}+\sum_{t=0}^{n} \frac{F F N t}{(k)^{t}}=\frac{[150000 ; 185000 ; 215000]}{[0.12 ; 0.15 ; 0.17]}+\frac{[160000 ; 200000 ; 230000]}{[0.12 ; 0.15 ; 0.17]^{2}}+$ $\frac{[173000 ; 205000 ; 230000]}{[0.15 ; 0.18 ; 0.2]^{3}}+\frac{[175000 ; 210000 ; 232000]}{[0.15 ; 0.18 ; 0.2]^{4}}+\frac{[180000 ; 212000 ; 235000]}{[0.15 ; 0.18 ; 0.2]^{5}}$

El cálculo se realiza de acuerdo con las operaciones definidas entre números difusos. El resultado es un número difuso que se interpreta en la forma adecuada.

\section{Jerarquía de riesgos}

Las empresas habitualmente desarrollan proyectos que están expuestos a riesgos. (Montero, 2015).

A continuación se presenta el siguiente ejemplo:

Una empresa desarrolla ciertos tipos de proyectos, los cuales precisa evaluar para determinar el riesgo más significativo. Del análisis que realiza infiere la existencia de tres riesgos, a cada uno de los cuales están asociados tres

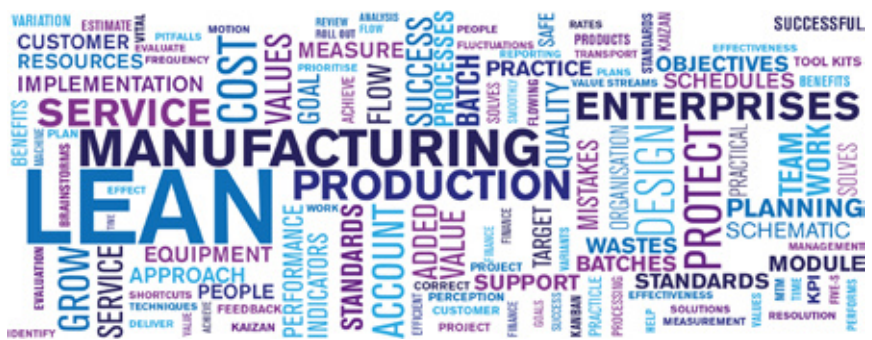

factores de incidencia.

Si se denomina por $Y$ el universo de los riesgos y por $X$ el universo de los factores que originan determinados riesgos, se tiene que $Y$ está conformado por los siguientes riesgos:

\section{$\mathrm{R} 1=$ personal \\ $\mathrm{R} 2$ = gestión \\ $\mathrm{R} 3=$ producto}

El producto cartesiano XxY está formado por los puntos que se interpretan como el factor que está influyendo en el riesgo correspondiente. Estos puntos son:
$(\mathrm{C} 1, \mathrm{R} 1)=$ dirección
$(\mathrm{C} 2, \mathrm{R} 1)=$ usuarios
$(\mathrm{C} 3, \mathrm{R} 1)=$ equipo de trabajo
$(\mathrm{C} 1, \mathrm{R} 2)=$ planificación
$(C 2, \mathrm{R} 2)=$ presupuesto
$(\mathrm{C} 3, \mathrm{R} 2)=$ ejecución
$(\mathrm{C} 1, \mathrm{R} 3)=$ calidad
$(\mathrm{C} 2, \mathrm{R} 3)$ = tecnología
$(\mathrm{C} 3, \mathrm{R} 3)=$ presentación

La función de pertenencia para estos puntos puede expresarse en forma tabular, como se indica en la tabla 1.

Hay varias estrategias aplicables a la selección del riesgo que se va a considerar más significativo: algunas desbalanceadas (max-max, min min), otras más conservadoras (máx.-mín, min -máx.). Una estrategia apropiada es la máx.-promedio, la cual está basada en un principio de razón insuficiente, por lo que asigna el mismo grado de posibilidad a las diversas alternativas.

\section{Toma de decisiones}

El modelo que aquí se muestra es una herramienta innovadora basada en la noción de la inferencia difusa y se aplica la metodología en el área de la industria turística. Como generalización de la implicación clásica entre proposiciones surge la noción de implicación entre conjuntos difusos que produce un nuevo conjunto difuso denominado cuya función de pertenencia es una función con ciertas propiedades que generalizan las de la función característica de la unión de conjuntos denominada t-conorma (Zadeh L., 1996). 
Por tanto, se puede generalizar la implicación entre los conjuntos difusos $\mathrm{P}$ y $\mathrm{A}$ dados por las funciones de pertenencia $f_{P}$ y $f_{A}$ respectivamente, definiéndola como un nuevo conjunto difuso denominado $\mathrm{P} \Rightarrow \mathrm{A}$ cuya función de pertenencia está dada en este caso por:

$$
f_{P \Rightarrow A}(c, a)=\min \left(1,1-f_{P}(c)+f_{A}(a)\right)
$$

Supongamos que se requiere determinar el mejor de los cuatro proyectos sobre un destino turístico, presentados por distintos turoperadores. Para esto, la agencia contacta con su departamento de consultoría para que le asesore y oriente en la selección del proyecto más adecuado.

Los especialistas han establecido siete condiciones o cualidades que les interesa medir y deben establecer un perfil o patrón conformado por las evaluaciones que entienden aceptables para cada una de las siete condiciones. Estas condiciones son: calidad de la oferta alimenticia (C1), calidad del alojamiento (C2), nivel de la oferta cultural (C3), variedad de la oferta para ratos de esparcimiento (C4), traslado interno (C5), traslado desde y hacia el aeropuerto (C6), servicio de guía (C7).

Para determinar en qué grado incide el perfil en la evaluación de cada condición para cada una de las ofertas se considera la función $f_{P \Rightarrow A}(c, a)$, donde $\mathrm{P}$ denota el conjunto de los valores del perfil para cada condición y $\mathrm{A}$ el conjunto de los proyectos.

La justificación para emplear esta fórmula está dada por su sentido objetivo:

Si $1-f_{P}(c)+f_{A}(a)$ es mayor que 1 , entonces $-f_{P}(c)+$ $f_{A}(a)$ es mayor que cero, es decir, $f_{P}(c)$ es menor que $f_{A}(a)$ y en este caso:

$$
f_{P \Rightarrow A}(c, a)=\min \left(1,1-f_{P}(c)+f_{A}(a)\right)=1
$$

Si $1-f_{P}(c)+f_{A}(a)$ es menor que 1 , entonces - $f_{P}(c)+$ $f_{A}(a)$ es menor que cero, es decir, $f_{P}(c)$ es mayor que $f_{A}(a)$ y en este caso

$$
f_{P \Rightarrow A}(c, a)=S_{L}\left(1-f_{P}(c), f_{A}(a)\right)=\min \left(1,1-f_{P}(c)+f_{A}(a)\right)
$$

es menor que 1.

El razonamiento anterior conduce a la siguiente interpretación:

Si la evaluación de la oferta en una condición es mayor o igual a la establecida por el patrón, entonces la evaluación correspondiente es máxima. Por tanto, se puede interpretar la función $f_{P \Rightarrow A}(c, a)$ como un indicador o coeficiente de adecuación de cada oferta a cada una de las diversas condiciones. Para obtener un valor global, estos valores se promediarán. El escalafón de las ofertas resultará del orden decreciente de estos números.

Los valores de $f_{P}(c)$ y $f_{A}(a)$ son datos que pueden manejarse en forma tabular, como se indica en la tabla 2, y los cálculos conformarse como se muestra en la tabla 3 , donde se aplica la fórmula que caracteriza la función de pertenencia.

\section{RESULTADOS Y DISCUSIÓN}

\section{Tabla 1: Evaluación de la función de pertenencia $f_{C}\left(\mathbf{C}_{\mathbf{i}}, \mathbf{R}_{\mathbf{k}}\right)$}

\begin{tabular}{|c|c|c|c|}
\hline Factor/Riesgo & $\mathrm{R}_{1}$ & $\mathrm{R}_{2}$ & $\mathrm{R}_{3}$ \\
\hline $\mathrm{C}_{1}$ & 0.3 & 0.4 & 0.1 \\
\hline $\mathrm{C}_{2}$ & 0.2 & 0.5 & 0.1 \\
\hline $\mathrm{C}_{3}$ & 0.7 & 0.35 & 0.75 \\
\hline
\end{tabular}

La aplicación de la estrategia máx.-promedio aporta los valores:

$g_{C}\left(R_{1}\right)=1 / 3\left[f_{C}\left(C_{1^{\prime}} R_{1}\right)+f_{C}\left(C_{2^{\prime}}, R_{1}\right)+f_{C}\left(C_{3^{\prime}} R_{1}\right)\right]=1 / 3(0.3+0.2+0.7)=0.4$

$g_{C}\left(R_{2}\right)=1 / 3\left[f_{C}\left(C_{1}, R_{2}\right)+f_{C}\left(C_{2^{\prime}} R_{2}\right)+f_{C}\left(C_{3^{\prime}} R_{2}\right)\right]=1 / 3(0.4+0.5+0.35)=0.416$

$g_{C}\left(R_{3}\right)=1 / 3\left[f_{C}\left(C_{1}, R_{3}\right)+f_{C}\left(C_{2^{\prime}} R_{3}\right)+f_{C}\left(C_{3^{\prime}} R_{3}\right)\right]=1 / 3(0.1+0.1+0.75)=0.316$

De aqui que:

Máx. $g_{c}(y)$

yєY

$=$ máx. $\left(f_{C}(\mathrm{R} 1), f_{C}(\mathrm{R} 2), f_{C}(\mathrm{R} 3), f_{C}(\mathrm{R} 4)\right)=$ máx. $(0.4,0.416,0.316)=0.416$

Por tanto, con esta estrategia se obtiene que el riesgo más significativo resulta el de gestión.

Tabla 2 Evaluación recibida por cada proyecto y el perfil establecido para cada cualidad.

\begin{tabular}{|c|c|c|c|c|c|}
\hline & $f_{C}\left(a_{1}\right)$ & $f_{C}\left(a_{2}\right)$ & $f_{C}\left(a_{3}\right)$ & $f_{C}\left(a_{4}\right)$ & $\begin{array}{c}\text { Perfil } \\
\left(f_{P}(c)\right)\end{array}$ \\
\hline C1 & 0 & 0.3 & 0.4 & 0.6 & 0.6 \\
\hline C2 & 0.9 & 0.8 & 0.8 & 1 & 0.9 \\
\hline C3 & 0.8 & 0.7 & 0.6 & 0.9 & 0.7 \\
\hline C4 & 1 & 0.4 & 1 & 0.5 & 1 \\
\hline C5 & 0.5 & 1 & 0.9 & 0.7 & 0.8 \\
\hline C6 & 0.4 & 0.9 & 0.3 & 0.5 & 0.3 \\
\hline C7 & 0 & 0.6 & 0.3 & 0.8 & 0.2 \\
\hline
\end{tabular}

Tabla 3: Cálculos obtenidos para la función de pertenencia.

\begin{tabular}{|c|c|c|c|c|}
\hline$f_{P \Rightarrow A}(c, a)$ & a1 & a2 & a3 & a4 \\
\hline$=\min \left(1,1-f_{P}(c)+f_{A}(a)\right)$ & 0.4 & 0.7 & 0.8 & 1 \\
\hline & 1 & 0.9 & 0.9 & 1 \\
\hline & 1 & 1 & 0.9 & 1 \\
\hline & 1 & 0.4 & 1 & 0.5 \\
\hline & 0.7 & 1 & 1 & 0.9 \\
\hline & 1 & 1 & 1 & 1 \\
\hline Promedio & 0.8 & 1 & 1 & 1 \\
\hline & 0.84 & 0.82 & 0.94 & 0.91 \\
\hline
\end{tabular}


De acuerdo con lo anterior, el escalafón sitúa la evaluación de las ofertas en el siguiente orden: 3,4, 1 y 2

Lo novedoso de esta investigación radica en la propuesta de utilizar las técnicas de la matemática difusa en la gestión de proyectos. Estas técnicas pueden aplicarse en los distintos momentos de la realización de un proyecto, según las situaciones problémicas que se vayan presentando en el desarrollo del mismo. Por esto, es importante que se conozcan de manera general para poder aplicarlas en el momento conveniente.

De lo expuesto con anterioridad, se deducen las siguientes formas de diseñar el análisis correspondiente a cada caso de gestión de proyectos tratado en esta investigación.

Respecto a la generación de índices para la valoración de la cuantía de un proyecto, se recomienda seguir la siguiente metodología:

1. Elegir la herramienta apropiada para problemas similares correspondiente a un ambiente de certidumbre.

2. Adaptar dicha herramienta al entorno de incertidumbre mediante la utilización de los números triangulares borrosos.

3. Interpretación apropiada del número difuso dado como salida del índice generado.

Respecto a la estimación del riesgo o jerarquización de los riesgos que se pueden presentar en el desarrollo de un proyecto, se propone:

1. Determinar los posibles riesgos y sus causas.

2. Relacionar por una función de pertenencia cada riesgo con sus causas.

3. Seleccionar la estrategia para la elección del mayor riesgo o la jerarquización de los distintos riesgos.

Para los problemas de toma de decisiones se sugiere proceder de la siguiente forma:

1. Definir la meta que se quiere lograr, y las alternativas posibles que responden a dicho objetivo.

2. Enunciar criterios que tributen al objetivo, destinados a la medición o comparación de las alternativas.

3. Decidir qué método jerárquico aplicar para la confección del ranking de alternativas.

\section{CONCLUSIONES}

Las técnicas derivadas de la lógica difusa son de gran importancia para la gestión de proyectos valorados en ambientes de incertidumbre.

Se propuso el uso de estas, tanto en la valoración de la cuantía del proyecto, en la estimación de riesgos, como en la toma de decisiones.
Se aprecia la utilidad de cada técnica mostrada, y consecuentemente la importancia de dar un tratamiento consecuente con la lógica difusa a los problemas presentados en los proyectos.

Se ejemplifica la utilización de esta importante rama de la matemática computacional en este sentido en varias áreas de aplicación social y económica relevante.

Se brinda una pauta metodológica a seguir para determinados problemas que pueden presentarse en los proyectos.

\section{BIBLIOGRAFÍA}

[1] Cruz Martínez, A. e. (2017). La lógica difusa en la modelización del riesgo operacional. Una solución desde la inteligencia artificial en la banca cubana. Cofin vol.11 no.2 La Habana jul.dic. , Cofin vol.11(2).

[2] Díaz Córdova, J. F., Coba Molina, E., \& Navarrete, P. (2017). Lógica difusa y el riesgo financiero. Una propuestade clasificación de riesgo financiero al sector cooperativo. Contaduría y Administración 62, 1670-1686.

[3] Grau, N., \&Bodea, C.-N. (2014). ISO 21500 project management standard: : Characteristics, comparison and implementation. VShaker Verlag GmbH, Germany.

[4] Institute, P. M. (2017). A guide to the project management body of knowledge (PMBOK guide) Sixth Edition / Project Management Institute. Project Management Institute (PMI), Inc. Newtown Square, Pennsylvania 19073-3299 USA.

[5] ISO. ISO 21500:2012 (2012) Guidance on Project Management. International Organization for Standardization. Disponible en: http://www.iso.org/iso/catalogue_ detail?csnumber $=50003$.

[6] Montero, J. C. (26 de noviembre de 2015). Aplicación de la lógica difusa compensatoria en el sector empresarial. Obtenido de Research Gate: www.researchgate.net/ publication/284722771_Aplicacion_de_la_logica_difusa_ compensatoria_en_el_sector_empresarial.

[7] Pacelli, L., (2004). The Project Management Advisor: 18 major project screw-ups, and how to cut them off at the pass. Pearson Education.

[8] Ruvalcaba Coyaso, F. J., \& Vermonden, A. ( 2015 ). Lógica difusa para la toma de decisiones y la selección de personal. Universidad \& Empresa, vol. 17, no. 29.

[9] Vostrov, M. (24 de enero de 2014). Ejemplo simple de creación de un indicador usando la lógica difusa. Obtenido de MQL5: www.mql5.com/es/articles/178

[10] Stellingwerf, R., \& Zandhuis, A. (2013). ISO 21500 Guidance On Project Management: A Pocket Guide (Best Practice). Van Haren.

[11] Zadeh, L. (1994). Fuzzy logic. Neural networks and soft computing. Communications of the ACM , 77-84.

[12] Zadeh, L. A. (1996). Fuzzy Sets, Fuzzy Logic, and Fuzzy Systems: Selected Papers. George Klir. 